\title{
Projected cancer risks potentially related to past, current, and future practices in paediatric CT in the United Kingdom, 1990-2020
}

\author{
Neige MY Journy ${ }^{\star}, 1$, Choonsik Lee ${ }^{1}$, Richard W Harbron ${ }^{2,3}$, Kieran McHugh ${ }^{4}$, Mark S Pearce ${ }^{2,3}$ \\ and Amy Berrington de González ${ }^{1}$
}

\begin{abstract}
${ }^{1}$ Radiation Epidemiology Branch, Division of Cancer Epidemiology and Genetics, National Cancer Institute, National Institutes of Health, 9609 Medical Center Drive, MSC 9776, Bethesda, Maryland 20892, USA; ${ }^{2}$ Institute of Health \& Society, Newcastle University, Royal Victoria Infirmary, Queen Victoria Road, Newcastle-upon-Tyne NE1 4LP, UK; ${ }^{3}$ NIHR Health Protection Research Unit in Chemical and Radiation Threats and Hazards, Newcastle University, Newcastle-upon-Tyne, UK and ${ }^{4}$ Great Ormond Street Hospital for Children, Great Ormond Street, London WC1N 3JH, UK
\end{abstract}

Background: To project risks of developing cancer and the number of cases potentially induced by past, current, and future computed tomography (CT) scans performed in the United Kingdom in individuals aged $<20$ years.

Methods: Organ doses were estimated from surveys of individual scan parameters and CT protocols used in the United Kingdom. Frequencies of scans were estimated from the NHS Diagnostic Imaging Dataset. Excess lifetime risks (ELRs) of radiation-related cancer were calculated as cumulative lifetime risks, accounting for survival probabilities, using the RadRAT risk assessment tool.

Results: In 2000-2008, ELRs ranged from 0.3 to 1 per 1000 head scans and 1 to 5 per 1000 non-head scans. ELRs per scan were reduced by 50-70\% in 2000-2008 compared with 1990-1995, subsequent to dose reduction over time. The 130750 scans performed in 2015 in the United Kingdom were projected to induce 64 (90\% uncertainty interval (UI): 38-113) future cancers. Current practices would lead to about 300 (90\% UI: 230-680) future cancers induced by scans performed in 2016-2020.

Conclusions: Absolute excess risks from single exposures would be low compared with background risks, but even small increases in annual CT rates over the next years would substantially increase the number of potential subsequent cancers.

Fifteen years ago, Brenner et al (2001) first assessed the possible magnitude of cancer risks induced by paediatric computed tomography (CT) and raised concerns about potential harmful effects of these X-ray exposures. That study predicted risks of fatal cancer ranging from 1 per 10000 to 1 per 1000 scanned patients, depending on their age and the scanned body part. Based on current radiological practices at that time in the United States, they projected that about 500 children scanned each year would ultimately die from a radiation-related cancer. Several investigators also reported the use of adult-calibrated scan parameters in paediatrics in this past period, resulting in unnecessarily high radiation doses in small body size patients (Mettler et al, 2000; Donnelly et al, 2001; Huda and Vance, 2007).

Since then, direct evidence of increased cancer risks after CT scans received in childhood or early adulthood has been provided in epidemiological studies (Pearce et al, 2012; Mathews et al, 2013; Huang et al, 2014; Journy et al, 2016), although there were uncertainties in the dose estimates and a possibility of bias owing to underlying medical conditions (Walsh et al, 2014; Berrington de Gonzalez et al, 2016). These studies have enhanced awareness about potential risks of X-ray exposures among the medical community and, along with considerable technological progress in

*Correspondence: Dr NMY Journy; E-mail: neige.journy@nih.gov

Received 24 June 2016; revised 12 September 2016; accepted 25 September 2016; published online 8 November 2016

(c) 2017 Cancer Research UK. All rights reserved 0007-0920/17 
CT, has led to further radiation dose optimisation in paediatrics. For instance, a survey in Great Britain showed that doses per scan were reduced by $50 \%$ in 2000-2008 compared with exposures before 1990 (Lee et al, 2016). At the same time, however, the number of examinations performed annually has considerably increased, in both adults and children (Pearce et al, 2011), owing to the more widespread availability of CT scanners, the considerable reduction in scan times (which now makes the use of sedation unnecessary in most children), and improvements in CT image quality allowing more medical applications. The increasing frequency of CT use has undoubtedly provided considerable medical benefits to children but at the same time has increased the collective radiation exposure and the number of possible radiationrelated cancers (Linton et al, 2003). In 2010, Parkin and Darby estimated that, in the United Kingdom, $0.6 \%$ of all cancers would be attributable to radiation exposures from diagnostic imaging in both paediatrics and adults (Parkin and Darby, 2011).

Our aim here is to estimate the potential radiation-related cancer risks from current CT practice in the United Kingdom, specifically in paediatrics, compared with past practice, and to quantify the impact of the documented dose reductions. We then use data on frequency of $\mathrm{CT}$ use to project the numbers of future cancers possibly attributable to paediatric scans currently performed in the United Kingdom, or will be in the next 5 years, in individuals aged $<20$ years.

\section{MATERIALS AND METHODS}

Projection of excess lifetime risks (ELRs) per scan. To project future cancer risks, we used the RadRAT risk assessment tool, which was developed at the National Cancer Institute, Bethesda, MD, USA (Berrington de Gonzalez et al, 2012), and is now freely accessible at https://irep.nci.nih.gov/radrat. RadRAT incorporates an extended list of cancer site-specific risk models that were previously derived by the US National Research Council in the BEIR VII report (NRC, 2006) from cohorts of survivors of the Hiroshima and Nagasaki atomic bombings and patients receiving radiotherapy for benign diseases or repeated diagnostic procedures. The above-mentioned recent studies on CT exposures cannot provide a full picture of radiation-related risks, mainly because their duration of follow-up is still too short to describe cancer incidence after the age of 50 years (Pearce et al, 2012; Mathews et al, 2013; Huang et al, 2014; Berrington de Gonzalez et al, 2016; Journy et al, 2016). In consequence, most of these studies estimated risks for a limited range of cancer sites. The risk estimates per unit dose were, however, compatible with the models implemented in RadRAT for leukaemia and cerebral tumours (no estimate per unit dose is available for other cancer sites), once children with previous cancers or cancer-predisposing conditions were excluded (Berrington de Gonzalez et al, 2016). Current evidence from CT scans thus provides support for the appropriateness of the BEIR VII/RadRAT models for our risk projection purposes.

From these models, ELRs of developing cancer were calculated for single CT scans, according to the patient's age at exposure, gender, and scanned body part, as cumulative risks that would occur in addition to baseline cancer risks (i.e., without CT exposure) over a lifetime, while accounting for survival probabilities at each attained age. Survival functions (England, 2011-2013) and baseline incidence rates (United Kingdom, 2011-2012) were obtained from the Office for National Statistics (ONS; www.ons.gov.uk, accessed on 26 March 2015). To account for risk projection uncertainties, $90 \%$ uncertainty intervals (UIs) were calculated as the 5th through to 95th percentile range of the distribution of ELR (or a total number of future cancers) values computed by Monte Carlo simulations using RadRAT. As detailed in the methodological paper, probability distributions were assigned to each of the following components of risk projection: dose-response model parameters, minimum latency period between radiation exposure and cancer occurrence, high-to-low doses risk extrapolation, and population-to-population risk transport, as well as to organ doses (see 'Organ dose per scan' section), to propagate uncertainties and dose variability in risk projection (Berrington de Gonzalez et al, 2012). All results on projected risks are displayed here as median simulated values with $90 \%$ UI.

Projection of total number of future cancers in the United Kingdom. The total number of future cancers potentially induced by annual frequencies of CT scans was calculated as a sum of estimated numbers of scans in a year for a given age group, gender, and scanned body part, multiplied by the corresponding ELRs. The presumed linear dose-response relationship for solid cancers and leukaemia over the dose range of our interest $(<0.5 \mathrm{~Gy}$; Preston et al, 2007; Wakeford, 2013) implies that the sum of projected risks for children who received multiple exposures is simply equal to the sum of projected risks per scan over all exposures in the population. The number of cancers potentially related to future scans for the period 2016-2020 was projected under different scenarios of dose reduction and future annual CT rates, which are detailed below. The total number of cancers potentially induced by past CT use (1990-2012) was not projected, owing to the lack of data on frequencies of paediatric CT scans in this time period, and the overly speculative nature of retrospective risk projections over such a long period.

Organ doses per scan. Organ doses were estimated by age at exposure $(0-4,5-9,10-14,15-19$ years), scanned body part (head, chest, abdomen-pelvis), gender, and time period (1990-1994, 1995-1999, 2000-2008), mainly from individual scan parameters extracted from a sample of 1073 procedures in members of the UK CT cohort (Lee et al, 2016), used to refine dosimetry since the first publication (Pearce et al, 2012). We converted the values of volume Computed Tomography Dose Index $\left(\mathrm{CTDI}_{\mathrm{vol}}\right)$ estimated from the scan parameters into organ doses using conversion coefficients and standard landmarks, as described previously (Lee et al, 2016). For risk projection, the variability in organ doses was described by lognormal distributions derived from the 1073 CT scan sample. For less frequently scanned body parts (cervical spine, shoulders, hips) and particular protocols (high-resolution, whole-body CT), data from the sample were very sparse. We thus used 'typical' $\mathrm{CTDI}_{\mathrm{vol}}$ values published from two national surveys of CT protocols used in the United Kingdom (Kim et al, 2012). For these infrequently scanned body parts, we did not account for dose variability (or uncertainty) in risk projection because no variability parameters were provided in the two national reference surveys. No dose estimation was performed for scans of the limbs because published values of $\mathrm{CTDI}_{\mathrm{vol}}$ were not reported separately for both legs and arms (Kim et al, 2012), and conversion factors were not developed for arms (Lee et al, 2012). For the period 2016-2020, we considered three scenarios of possible future dose reduction (constant, $-20 \%$, or $-40 \%$, as compared with doses per scan in 2000-2008), which would result from technological innovation and improved dose optimisation (Dougeni et al, 2012; Raman et al, 2013).

Frequencies of paediatric CT scans in the United Kingdom. The total number of scans in England in 2013-2015 was collected from the Diagnostic Imaging Dataset, which gathers information about all imaging tests carried out in England through the National Health Service (NHS) since April 2012 (reports accessible at www.england.nhs.uk). Data were obtained by 5 -year age group and gender, excluding CT-guided procedures (e.g., biopsies or drainage), which are usually associated with very small doses. Procedures with unknown age or gender $(<3 \%)$ were assumed to 
have the same age and gender distribution as scans with specified patients' age and gender and were added to subtotals. To estimate the number of scans by body part, we applied frequencies by scan type and 5-year age groups estimated in the UK CT cohort (Pearce et al, 2012). The total number of scans throughout the United Kingdom was estimated by applying CT rates per 1000 inhabitants derived from NHS England data by 5-year age group and calendar year to the 2012-based population projections for the whole of the United Kingdom published by the ONS (http://www.ons.gov.uk, accessed on 7 March 2016). In the United Kingdom, paediatric CT scans are virtually all performed within the public NHS system. The number of CT scans performed over the next 5 years was then projected under various realistic scenarios of future changes in annual CT rates per inhabitant (constant, $+5,+3$, or $-2 \%$, as compared with the rates in 2015), and a 'worst case' scenario of annual increase by $10 \%$ corresponding to CT trends observed in past years in other countries (Smith-Bindman et al, 2012; Brady et al, 2016; Dovales et al, 2016).

\section{RESULTS}

ELRs associated with single CT scans in 1990s and 2000s. The projected ELR per scan decreased by $50-70 \%$ during the period 2000-2008 compared with the period 1990-1994, depending on age at exposure, gender and scanned body part (Figure 1). In 20002008, ELRs ranged from 0.3 to 1 per 1000 head scans and 1 to 5 per 1000 non-head scans (scans of the chest or abdomen and pelvis) according to patient's gender and age (Table 1). For head scans, projected ELRs were similar for both genders, but for non-head scans, ELRs were 1.5-3 times higher in girls than in boys owing to higher risks of thyroid, breast, lung, and gynaecological cancers. As compared with a background lifetime risk of $40 \%$ in unexposed children, each single scan during childhood would lead to one excess case per 1000 spontaneous cancers, on average. Uncertainties in risk projection were nevertheless large, for example, for chest scans in girls aged 5 years, $90 \%$ of the simulated ELR values ranged from 1 to 13 per 1000 (Table 1).

Number of future cancers potentially induced by scans performed in 2015. In England, the annual rate of CT use increased by $3 \%$ on average over the period $2013-2015$ up to 8.5 scans per 1000 in 2015 . The 2015 rates were, respectively, 5.6, 3.8, 7.0, and 17.9 per 1000 in individuals aged $0-4,5-9,10-14$, and $15-19$ years. Based on these figures, we projected that 64 (90\% UI: 38-113) future cancers would be induced by the 130750 scans performed in 2015 in the United Kingdom in individuals aged $<20$ years. Girls accounted for $46 \%$ of the projected future cancers; adolescents aged 15-19 years accounted for half and infants ( $<1$-year-old at scan) for almost $10 \%$ of projected future cancers (Figure 2). Cerebral tumours were the most frequent potentially radiation-related cancers, accounting for a fourth of all projected future cancers (Figure 3). Leukaemia, oral, lung, breast, and colorectal cancers accounted each for 1 out of 10 . Despite the fact that cervical spine, chest, abdomen, and/or pelvis scans accounted for only one-fifth of all examinations, tumours of organs located exclusively in the neck, thoracic, or abdominal region (thyroid gland, breasts, lungs, digestive, and urinary organs) accounted for half of all projected future cancers, owing to their high sensitivity to radiation.

Projected future cancers from different scenarios of CT practices up to 2020. While considering the doses per scan during the period 2000-2008 and the CT rates of 2015 remaining constant up to 2020, we calculated that 320 (90\% UI: 230-680) future cancers would be induced by paediatric CT use in the United Kingdom over the next 5 years (Table 2). If the frequency of scans continues to increase by $3 \%$ per year up to 2020 , this would lead to a number of potential future cancers increasing by $10 \%$, as compared with constant CT rates. Rates increasing annually by $5 \%$ and $10 \%$ would be associated with increasing numbers of subsequent future cancers by $16 \%$ and $34 \%$, respectively, by 2020 . Countering these projections are further CT dose reduction techniques likely to be developed or implemented in the future, which would proportionally decrease the associated potential cancer risks (Table 2).

\section{DISCUSSION}

This study is an updated risk assessment for paediatric CT scans, which accounts for recent trends in radiation doses and frequency of use in the United Kingdom. Compared with the earliest period of CT use (before 1995), it shows that potential cancer risks per scan have been reduced by $50-70 \%$ in recent years owing to dose reduction practices over time. With an estimated annual rate of 8.5 scans per 1000 children and adolescents, resulting in a total of 131000 scans in 2015, we projected that 40-110 children who were scanned in that year would ultimately develop a radiation-related cancer over their lifetime. To put this in context, if we assume that 110000 children were scanned that year (1.2 scan per child on average), 44000 of these children would develop a cancer during their life, independently of their CT exposure in childhood (assuming a background lifetime risk of cancer of 40\%).

An rough estimate of 5 future cancers per 10000 paediatric scans from current practices in the United Kingdom is substantially lower than in previous studies conducted from past CT practices in the United States, which estimated 8 cancer deaths (Brenner et al, 2001) and 10-12 incident cancers (Berrington de Gonzalez et al, 2009; Miglioretti et al, 2013) attributable to 10000 paediatric scans. A reduction of risks per scan in the United States by a similar extent to our estimates for the United Kingdom would be especially meaningful in terms of cancer burden reduction in this larger population. A projection rate of 5 future cancers per 10000 scans would correspond to 3400 future cancers possibly induced by the 6.8 million paediatric scans performed in the United States in 2014 (IMV, 2014), as compared with the 70008000 future cancers that we project from the previous estimates (Berrington de Gonzalez et al, 2009; Miglioretti et al, 2013). This reduction assumes that the reduced doses per scan observed in the United Kingdom also apply in the United States, although, to our knowledge, no large-scale survey has described very recent trends in radiation doses in routine paediatric care in the United States. Transposing current results for the United Kingdom to another population also assumes that the two populations have similar background risks and distribution of age at scan and scanned body parts, which can be considered as a reasonable assumption (Ferlay et al, 2012).

Other previous studies have projected cancer risks from paediatric CT scans. Most of them have reported radiation exposure and potential subsequent risks based on dedicated CT protocols, for example, for monitoring of cystic fibrosis (de Jong et al, 2006), detection of renal calculi (Kuhns et al, 2011), treatment of neurovascular diseases (Raelson et al, 2009), low-dose chest scan (Niemann et al, 2015), or coronary angiography (Huang et al, 2009). Few other studies have projected risks per scan from standard paediatric CT protocols, for example, in the United States (Li et al, 2011), France (Journy et al, 2014), and China (Su et al, 2014). None, to our knowledge, has previously considered routine practices in the United Kingdom. Many components differ between these studies, including population-specific background cancer risks and life expectancy, organ doses (scan parameters including the length of the scan region, and methods to estimate organ doses), risk models (though relatively homogeneous models were used in most studies), and methods to propagate risk 

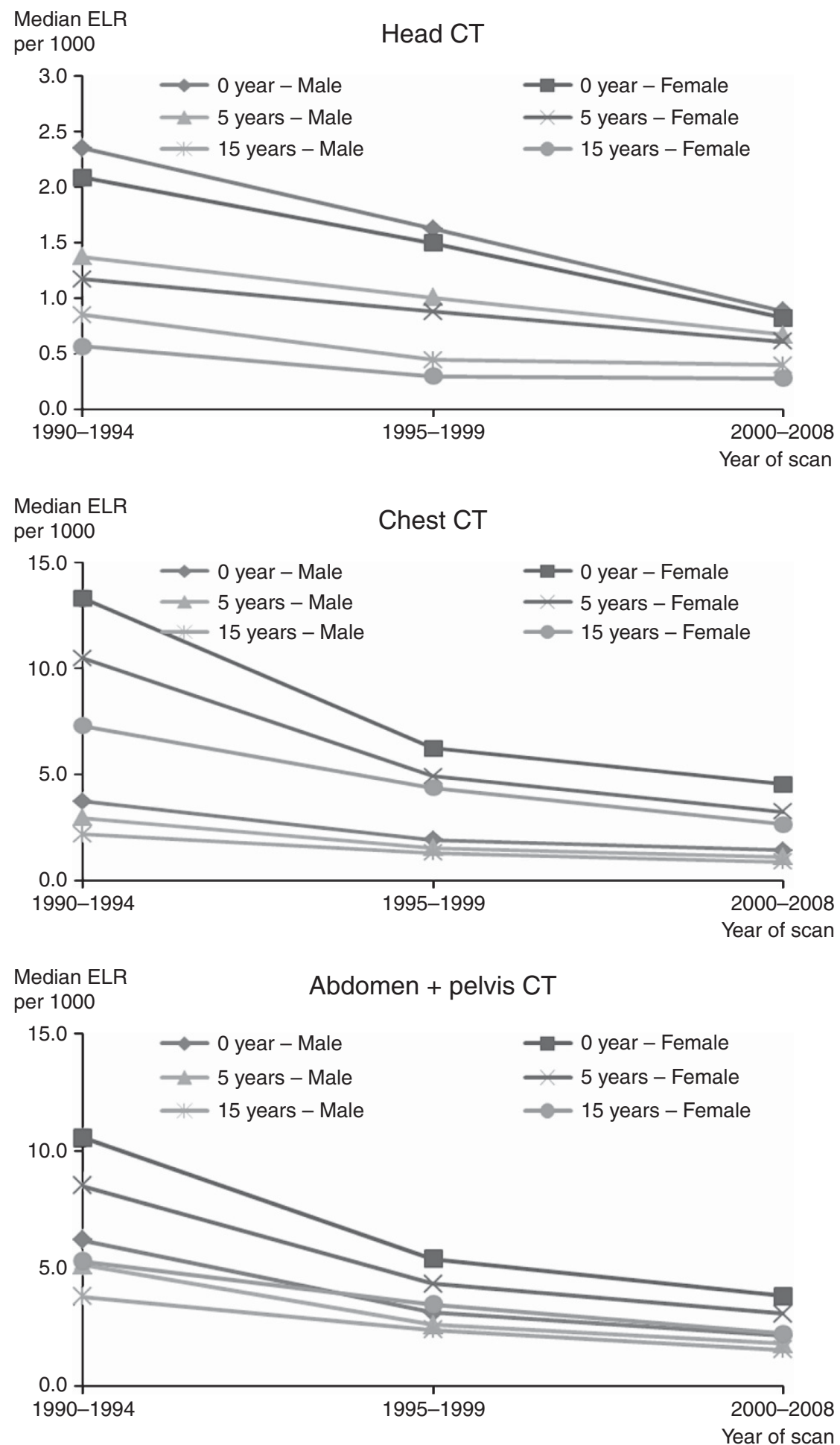

Figure 1. Projected ELR scans for the period 1990-2008.

projection uncertainties. However, our projection of $0.1-1$ incident case (all cancer sites combined) per 1000 head scans and 1-5 per 1000 non-head scans is completely consistent with other studies reporting all-cancer risks from standard CT protocols (Berrington de Gonzalez et al, 2009; Li et al, 2011; Miglioretti et al, 2013). With the use of largely similar risk models, these studies also consistently reported 2-7 times higher risks per scan in girls than in boys (for non-head scans only) and 1.5-3 times higher risks per scan in neonates than in adolescents aged 10-15 years (for all scans)
(Berrington de Gonzalez et al, 2009; Li et al, 2011; Miglioretti et al, 2013; Journy et al, 2014).

The current study benefited from the use of empirical data on radiation doses and frequency of exposure in the United Kingdom for the past and current time periods. The source of information used for organ dose estimation is the only one to include both individual variability and temporal trends from 1990 to 2008 in the United Kingdom and will be part of a refined dosimetry of the UK CT cohort (Lee et al, 2016). Since the most recent period (2000- 
2008) of this survey, progress in CT technology and improved practices in dose optimisation are likely to have reduced radiation doses that are currently delivered to patients and to reduce them even more in the future. Although future technological advances are unpredictable, we assessed different scenarios of dose reduction compatible with the expected gains from the widespread use of recent technological innovations (particularly automatic exposure control and iterative reconstruction) (Dougeni et al, 2012; Raman et al, 2013). A recent national survey in the United Kingdom, however, suggested few changes in paediatric CT practices in 2011 compared with 2003 (PHE, 2014). Future frequencies of CT use are also unpredictable, but 'realistic' scenarios of future annual CT rates increasing by $-2 \%$ to $+5 \%$ would predict total numbers of possibly induced future cancers varying from $-6 \%$ to $+16 \%$ in the United Kingdom by the end of 2020, as compared with CT rates in 2015. A 'worst case scenario' of annual rates increasing by $10 \%$ up to 2020 would lead to an increased total number of potential subsequent cancers by $34 \%$ in the next 5 years.

As has been extensively discussed in the literature (NRC, 2006; UNSCEAR, 2012), the methodological framework for low-dose radiation risk projection has several limitations. In our particular context, the main sources of uncertainty are related to the shape of the dose-response relationship, particularly at low doses $(<0.1 \mathrm{~Gy})$, the joint effect of radiation and other risk factors for cancer, the existence of modifying effects, such as age at exposure, and the latency time between radiation exposure and cancer diagnosis. Propagation of uncertainties through Monte Carlo simulations as implemented in RadRAT accounts for most of these sources of uncertainties to provide ranges of possible risk values. However, RadRat only considers one set of no-threshold risk models and does not consider different shapes of the doseresponse relationship and effects of modifying effects (Berrington

Table 1. Projected excess lifetime risk (ELR) and uncertainty intervals of all cancers incidence per $1000 \mathrm{CT}$ scans, according to the time period of scan, the scanned body part, and patient's gender and age

\begin{tabular}{|c|c|c|c|c|c|c|c|c|}
\hline \multirow[b]{2}{*}{ Scanned body part } & \multirow[b]{2}{*}{$\begin{array}{c}\text { Age } \\
\text { (in years) }\end{array}$} & \multirow[b]{2}{*}{ Gender } & \multicolumn{3}{|c|}{$\begin{array}{c}\text { ELR ( } 90 \% \text { uncertainty interval) } \\
\text { by time period }\end{array}$} & \multicolumn{3}{|c|}{$\begin{array}{l}\text { Relative difference between } \\
\text { time periods, }(\%)^{a}\end{array}$} \\
\hline & & & $\begin{array}{l}\text { 1990-1994 } \\
\text { (1) }\end{array}$ & $\begin{array}{l}1995-1999 \\
\text { (2) }\end{array}$ & $\begin{array}{c}2000-2008 \\
\text { (3) }\end{array}$ & (2) vs (1) & (3) vs (2) & (3) vs (1) \\
\hline Head & \begin{tabular}{c|}
0 \\
5 \\
15
\end{tabular} & $\begin{array}{c}\text { Male } \\
\text { Female } \\
\text { Male } \\
\text { Female } \\
\text { Male } \\
\text { Female }\end{array}$ & $\begin{array}{l}2.4(1.2-4.8) \\
2.1(1.0-4.0) \\
1.4(0.7-2.7) \\
1.2(0.6-2.0) \\
0.9(0.4-1.8) \\
0.6(0.3-1.0)\end{array}$ & $\begin{array}{l}1.6(0.6-4.1) \\
1.5(0.7-3.2) \\
1.0(0.4-2.7) \\
0.9(0.4-1.8) \\
0.4(0.2-1.3) \\
0.3(0.1-0.8)\end{array}$ & $\begin{array}{l}0.9(0.4-2.3) \\
0.8(0.4-2.0) \\
0.7(0.3-1.7) \\
0.6(0.3-1.3) \\
0.4(0.2-1.2) \\
0.3(0.1-0.6)\end{array}$ & $\begin{array}{l}-33 \\
-29 \\
-29 \\
-25 \\
-56 \\
-50\end{array}$ & $\begin{array}{r}-44 \\
-47 \\
-30 \\
-33 \\
00 \\
00\end{array}$ & $\begin{array}{l}-63 \\
-62 \\
-50 \\
-50 \\
-56 \\
-50\end{array}$ \\
\hline Chest & $\begin{array}{r}0 \\
5 \\
15\end{array}$ & $\begin{array}{c}\text { Male } \\
\text { Female } \\
\text { Male } \\
\text { Female } \\
\text { Male } \\
\text { Female }\end{array}$ & $\begin{aligned} 3.7 & (1.8-8.7) \\
13.3 & (6.5-24.8) \\
3.0 & (1.4-7.1) \\
10.5 & (5.0-18.3) \\
2.2 & (1.2-4.3) \\
7.3 & (4.2-11.4)\end{aligned}$ & $\begin{array}{l}1.9(0.8-5.0) \\
6.3(2.7-16.3) \\
1.5(0.7-4.1) \\
4.9(2.1-12.6) \\
1.3(0.6-3.2) \\
4.4(1.9-8.8)\end{array}$ & $\begin{array}{l}1.4(0.5-6.0) \\
4.5(1.5-16.5) \\
1.1(0.4-4.9) \\
3.3(1.1-12.8) \\
0.9(0.4-2.1) \\
2.6(1.3-6.4)\end{array}$ & $\begin{array}{l}-49 \\
-53 \\
-50 \\
-53 \\
-41 \\
-40\end{array}$ & $\begin{array}{l}-26 \\
-29 \\
-27 \\
-33 \\
-31 \\
-41\end{array}$ & $\begin{array}{l}-62 \\
-66 \\
-63 \\
-69 \\
-59 \\
-64\end{array}$ \\
\hline
\end{tabular}

A Number of CT scans in 2015 All ages : 130750 scans

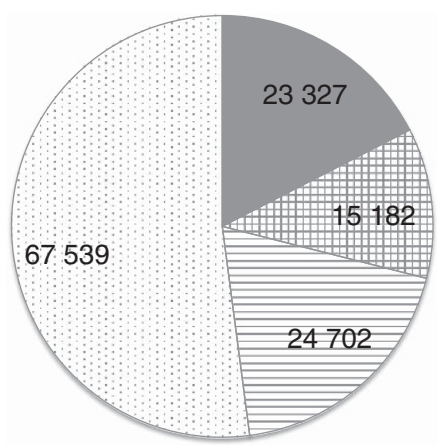

Age at scan (in years) $\quad$ - $0-4$
B Number of future cancers potentially induced All ages : 64 future cancers

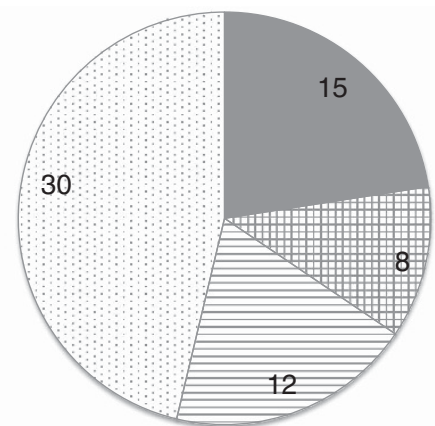

田 5-9 目 10-14 অ15-19

NB: Figures are reported only for diagnostic scans; they exclude CT-guided procedures

Figure 2. Pie chart of the usage of paediatric CT scans by age group. (A) Number of scans performed in the UK in 2015. (B) Number of future cancers potentially induced by those scans. 
de Gonzalez et al, 2012). Using the same reference data as used to estimate the BEIR VII/RadRAT models, other risk models have been preferred by other authors and scientific committees, in particular the United Nations Scientific Committee on the Effects of Atomic Radiation (UNSCEAR, 2006) and US Environmental Protection Agency (EPA, 2011). These models generally use different assumptions to model the dose-response relationship and account for confounding factors and effect modifiers. Attempts have been made to develop methods to account for model uncertainty based on goodness-of-fit criteria, but they might lead to omitting important confounding factors (Richardson and Cole, 2012) or effect modifiers (e.g., age at exposure), which could be critical for risk projection purposes.

Extrapolation of risks from moderate-to-high doses (0.1 to $>2 \mathrm{~Gy})$ to the low-dose range $(<0.1 \mathrm{~Gy})$ of single CT exposures remains controversial (Doss, 2013). CT scan studies do not have a sufficient follow-up to provide risk estimates over a lifetime and for cancer sites that usually occur at old ages (e.g., thyroid, breast, lung, digestive cancers). These studies are nevertheless helpful to assess the validity of BEIR VII/RadRAT models (or others) for risk projection purposes. First analyses in the UK and the Australian studies showed risk estimates for brain tumours that were higher than what the BEIR VII/RadRAT risk models would have predicted, with an excess relative risk (ERR) per mGy of 0.02 (95\% CI: $0.01-0.04)$ vs 0.006 (95\% CI: 0-0.06) in the a-bomb survivors who were exposed before age 20 years and followed up to 20 years after exposure (Pearce et al, 2012; Mathews et al, 2013). However, a further analysis of the UK CT cohort showed that the risk estimates were reduced, though remaining significantly increased, after excluding children with a previous unreported

\section{A Body parts scanned by CT}

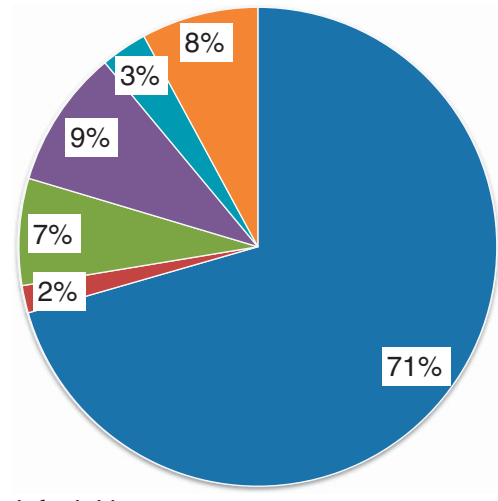

- Head, facial bones

- Cervical spine

Chest

- Abdomen \pm pelvis

- Pelvis

Extremities, hips, shoulders, whole body
B Projected future cancers

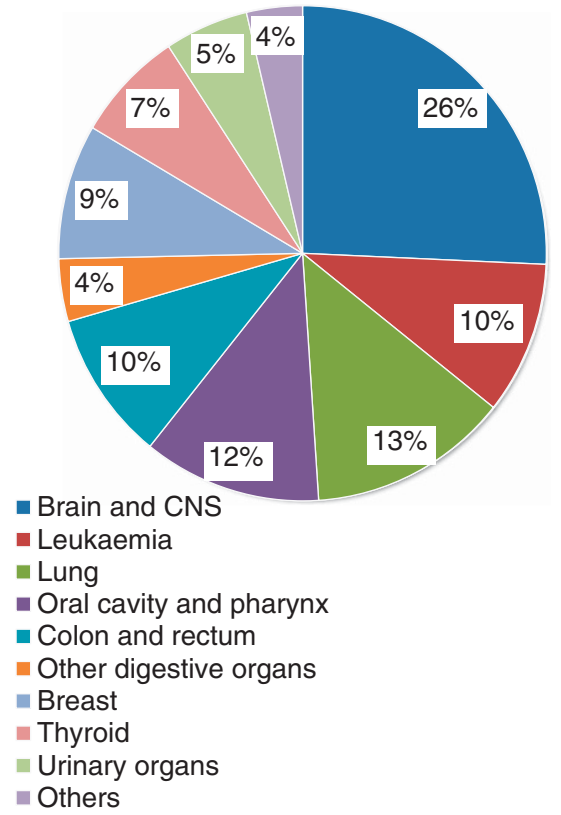

Figure 3. Pie chart of the usage of paediatric CT scans by scanned body part. (A) Body parts scanned by CT. (B) Projected future cancers by tumour site. CNS = central nervous system. NB: Figures are reported only for diagnostic scans; they exclude CT-guided procedures.

Table 2. Projected number of future cancers potentially induced by CT scans performed over the next 5 years (period 20162020 ) in the United Kingdom, according to various scenarios of future practices in paediatrics

\begin{tabular}{|c|c|c|c|c|}
\hline & & & \multicolumn{2}{|c|}{$\begin{array}{l}\text { Avoided or additional future cancers as } \\
\text { compared with the reference scenario }\end{array}$} \\
\hline $\begin{array}{l}\text { Change in doses per scan as } \\
\text { compared with practices in } \\
2000-2008\end{array}$ & $\begin{array}{l}\text { Change in annual CT } \\
\text { rate per inhabitant as } \\
\text { compared with } 2015\end{array}$ & $\begin{array}{c}\text { Projected future cancers } \\
\text { potentially induced by scans } \\
\text { performed in 2016-2020 ( } 90 \% \\
\text { uncertainty interval) }\end{array}$ & Absolute difference & Relative difference \\
\hline Dose remaining constant & $\begin{array}{l}+10 \% \\
+5 \% \\
+3 \% \\
+0 \% \\
-2 \%\end{array}$ & $\begin{array}{l}430(300-870) \\
370(260-770) \\
350(250-730) \\
320(230-680) \\
300(220-650)\end{array}$ & $\begin{array}{l}+111 \\
+52 \\
+30 \\
\quad \text { Referenc } \\
-19\end{array}$ & $\begin{array}{ll} & +34 \% \\
& +16 \% \\
& +9 \% \\
& \\
& \\
& -6 \%\end{array}$ \\
\hline $20 \%$ reduction & $\begin{array}{l}+10 \% \\
+3 \% \\
+0 \%\end{array}$ & $\begin{array}{l}350(240-700) \\
280(200-590) \\
260(180-540)\end{array}$ & $\begin{array}{l}24 \\
-40 \\
-64\end{array}$ & $\begin{array}{l}+7 \% \\
-12 \% \\
-20 \%\end{array}$ \\
\hline $40 \%$ reduction & $\begin{array}{l}+10 \% \\
+3 \% \\
+0 \%\end{array}$ & $\begin{array}{l}260(180-520) \\
210(150-440) \\
190(140-410)\end{array}$ & $\begin{array}{l}-62 \\
-111 \\
-129\end{array}$ & $\begin{array}{l}-19 \% \\
-34 \% \\
-40 \%\end{array}$ \\
\hline
\end{tabular}


diagnosis of cancer or suspected tumour at the time of scan, with an ERR per mGy of 0.01 (95\% CI: 0.004-0.03; Berrington de Gonzalez et al, 2016). After accounting for indication bias, this latest analysis thus provides risk estimates that are compatible with the results from the a-bomb survivors' study, especially if we consider that a residual indication bias may remain in the CT risk estimates. The risk estimates for leukaemia, including myelodysplasia, after CT exposures (ERRs per mGy comprised between 0.03 and 0.04 depending on the population considered) appeared also consistent with the results of the a-bomb survivor study (ERR per $\mathrm{mGy}=0.04$; Pearce et al, 2012; Mathews et al, 2013; Berrington de Gonzalez et al, 2016), which also includes cases of myelodysplasia during the early follow-up (Hsu et al, 2013). Current evidence from CT scan studies thus support the appropriateness of the BEIR VII/ RadRAT models to evaluate potential risks subsequent to CT exposures at a population level. At the current time, this conclusion is, however, limited to brain tumours and leukaemia only, as no dose-response analyses has been conducted to date for other cancer sites in studies with sufficient sample size. We thus acknowledge the need for further analyses with longer duration follow-up to fully address the issue of risk extrapolation to CT exposures.

Finally, caution is required to avoid interpreting the current results as individual risks. As discussed below, large uncertainties exist when projecting risks from one particular situation of radiation exposure to another one, and we must acknowledge that the sensitivity to radiation varies according to individual factors, such as genetic susceptibility or other cancer risk factors (UNSCEAR, 2013). In addition, we considered here that the current background cancer risks and life expectancy of the general UK population applied in children who receive CT scans, without considering temporal changes and individual underlying medical conditions that may impact the risk of cancer and survival. A reduced survival probability will obviously reduce the risk of radiation-related effects over a lifetime (Brenner et al, 2011; Harbron et al, 2016). The current results should therefore only be interpreted at the population level to provide a sense of the magnitude of the potential risks and impact of collective exposures and to assess possible future scenarios of CT practices. At the individual level, with potential absolute risks subsequent to CT exposures usually very low compared with the background lifetime risks of cancer, the immediate benefits of $\mathrm{CT}$, as currently utilised in the United Kingdom, would largely outweigh the risks in most clinical situations.

\section{CONCLUSION}

Changes in practice have substantially reduced the radiation doses to children from CT scans in the United Kingdom and potential subsequent cancer risks. However, the accompanying increase in frequency of scans has increased the collective exposure and the potential associated cancer burden. We estimated that about 230-680 future cancers would be induced by scans performed in children during 2016-2020 in the United Kingdom, if the frequency of CT use remains unchanged and no substantial further dose reduction occurs on a widespread scale. The absolute excess risk related to one CT scan would be very low as compared with background cancer risks that patients would face over their lifetime. Therefore, when paediatric CT is justified for a particular indication and other imaging tests are not adapted or available, the expected benefits of CT for children would largely outweigh the risks. However, because of the potential harmful effects of radiation exposure, paediatric CT scans need to be used in accordance with clinical guidelines and with proper dose optimisation to avoid unnecessary exposures and risks.

\section{ACKNOWLEDGEMENTS}

This study used data from the Diagnostic Imaging Dataset released by the National Health Service (NHS) England. We thank the NHS England for providing us detailed data on frequencies of paediatric CT scans. The interpretation and reporting of these data are the sole responsibility of the authors and do not necessarily reflect the view of the NHS. Likewise, the views expressed by the authors do not necessarily reflect those of the National Institute for Health Research (NIHR), the Department of Health or Public Health England, to which MSP and RWH are affiliated or collaborate with. The estimated frequencies of CT scans provided in this paper might differ from official statistics because we applied our own methodology to handle missing data on patients' characteristics. This study was financially supported by the US National Cancer Institute intramural research program, the UK Department of Health, and Cancer Research UK. The funding sources and sponsor had no role in design and conduct of the study; collection, management, analysis, and interpretation of the data; preparation, review, or approval of the manuscript; and decision to submit the manuscript for publication.

\section{CONFLICT OF INTEREST}

The authors declare no conflict of interest.

\section{REFERENCES}

Berrington de Gonzalez A, Iulian Apostoaei A, Veiga LH, Rajaraman P, Thomas BA, Owen Hoffman F, Gilbert E, Land C (2012) RadRAT: a radiation risk assessment tool for lifetime cancer risk projection. J Radiol Prot 32: 205-222.

Berrington de Gonzalez A, Mahesh M, Kim K-P, Bhargavan M, Lewis R, Mettler F, Land C (2009) Projected cancer risks from computed tomographic scans performed in the United States in 2007. Arch Intern Med 169: 2071-2077.

Berrington de Gonzalez A, Salotti JA, Mchugh K, Little MP, Harbron RW, Lee C, Ntowe E, Braganza MZ, Parker L, Rajaraman P, Stiller C, Stewart DR, Craft AW, Pearce MS (2016) Relationship between paediatric CT scans and subsequent risk of leukaemia and brain tumours: assessment of the impact of underlying conditions. Br J Cancer 114: 388-394.

Brady Z, Forsythe AV, Mathews JD (2016) The changing use of pediatric CT in Australia. Pediatr Radiol 46: 1199-1208.

Brenner D, Elliston C, Hall E, Berdon W (2001) Estimated risks of radiationinduced fatal cancer from pediatric CT. AJR Am J Roentgenol 176: 289-296.

Brenner DJ, Shuryak I, Einstein AJ (2011) Impact of reduced patient life expectancy on potential cancer risks from radiologic imaging. Radiology 261: 193-198.

de Jong PA, Mayo JR, Golmohammadi K, Nakano Y, Lequin MH, Tiddens HA, Aldrich J, Coxson HO, Sin DD (2006) Estimation of cancer mortality associated with repetitive computed tomography scanning. Am J Respir Crit Care Med 173: 199-203.

Donnelly LF, Emery KH, Brody AS, Laor T, Gylys-Morin VM, Anton CG, Thomas SR, Frush DP (2001) Minimizing radiation dose for pediatric body applications of single-detector helical CT: strategies at a large Children's Hospital. AJR Am J Roentgenol 176: 303-306.

Doss M (2013) Linear no-threshold model vs. radiation hormesis. Dose Response 11: 480-497.

Dougeni E, Faulkner K, Panayiotakis G (2012) A review of patient dose and optimisation methods in adult and paediatric CT scanning. Eur J Radiol 81: e665-e683.

Dovales AC, Da Rosa LA, Kesminiene A, Pearce MS, Veiga LH (2016) Patterns and trends of computed tomography usage in outpatients of the Brazilian public healthcare system, 2001-2011. J Radiol Prot 36: 547-560. 
EPA (2011) Radiogenic Cancer Risk Models and Projections for the US Population. US Environmental Protection Agency: Washington, DC, USA.

Ferlay J, Soerjomataram IHGIFPRA, Ervik M, Dikshit R, Eser S, Mathers C, Rebelo M, Parkin D, Forman D, Bray F (2012) GLOBOCAN 2012 v1.0, Cancer Incidence and Mortality Worldwide: IARC CancerBase No. 11 [Internet]. International Agency for Research on Cancer: Lyon, France 2013 Available from http://globocan.iarc.fraccessed on 26 August 2016.

Harbron RW, Chapple CL, O'Sullivan JJ, Best KE, Berrington de Gonzalez A, Pearce MS (2016) Survival adjusted cancer risks attributable to radiation exposure from cardiac catheterisations in children. Heart; e-pub ahead of print 18 August 2016; doi:10.1136/heartjnl-2016-309773.

Hsu WL, Preston DL, Soda M, Sugiyama H, Funamoto S, Kodama K, Kimura A Kamada N, Dohy H, Tomonaga M, Iwanaga M, Miyazaki Y, Cullings HM, Suyama A, Ozasa K, Shore RE, Mabuchi K (2013) The incidence of leukemia, lymphoma and multiple myeloma among atomic bomb survivors: 1950-2001. Radiat Res 179: 361-382.

Huang B, Law MW, Mak HK, Kwok SP, Khong PL (2009) Pediatric 64-MDCT coronary angiography with ECG-modulated tube current: radiation dose and cancer risk. AJR Am J Roentgenol 193: 539-544.

Huang WY, Muo CH, Lin CY, Jen YM, Yang MH, Lin JC, Sung FC, Kao CH (2014) Paediatric head CT scan and subsequent risk of malignancy and benign brain tumour: a nation-wide population-based cohort study. $\mathrm{Br} \mathrm{J}$ Cancer 110: 2354-2360.

Huda W, Vance A (2007) Patient radiation doses from adult and pediatric CT. AJR Am J Roentgenol 188: 540-546.

IMV (2014) CT Benchmark Report, 2014 Edition. IMV Medical Information Division, Inc. Des Plaines: Illinois, USA 22 pp.

Journy N, Ancelet S, Rehel JL, Mezzarobba M, Aubert B, Laurier D, Bernier MO (2014) Predicted cancer risks induced by computed tomography examinations during childhood, by a quantitative risk assessment approach. Radiat Environ Biophys 53: 39-54.

Journy N, Roué T, Cardis E, Le Pointe HD, Brisse H, Chateil JF, Laurier D, Bernier MO (2016) Childhood CT scans and cancer risk: impact of predisposing factors for cancer on the risk estimates. J Radiol Prot 36: N1-N7.

Kim KP, Berrington de Gonzalez A, Pearce MS, Salotti JA, Parker L, Mchugh K, Craft AW, Lee C (2012) Development of a database of organ doses for paediatric and young adult CT scans in the United Kingdom. Radiat Prot Dosimetry 150: 415-426.

Kuhns LR, Oliver WJ, Christodoulou E, Goodsitt MM (2011) The predicted increased cancer risk associated with a single computed tomography examination for calculus detection in pediatric patients compared with the natural cancer incidence. Pediatr Emerg Care 27: 345-350.

Lee C, Kim KP, Long DJ, Bolch WE (2012) Organ doses for reference pediatric and adolescent patients undergoing computed tomography estimated by Monte Carlo simulation. Med Phys 39: 2129-2146.

Lee C, Pearce MS, Salotti JA, Harbron RW, Little MP, Mchugh K, Chapple CL, Berrington de Gonzalez A (2016) Reduction in radiation doses from paediatric CT scans in Great Britain. Br J Radiol 89: 20150305.

Li X, Samei E, Segars WP, Sturgeon GM, Colsher JG, Toncheva G, Yoshizumi TT, Frush DP (2011) Patient-specific radiation dose and cancer risk estimation in CT: Part II. Application to patients. Med Phys 38: 408-419.

Linton OW, Mettler Jr FA. National Council On Radiation P and Measurements (2003) National conference on dose reduction in CT, with an emphasis on pediatric patients. AJR Am J Roentgenol 181: 321-329.

Mathews JD, Forsythe AV, Brady Z, Butler MW, Goergen SK, Byrnes GB, Giles GG, Wallace AB, Anderson PR, Guiver TA, Mcgale P, Cain TM, Dowty JG, Bickerstaffe AC, Darby SC (2013) Cancer risk in 680000 people exposed to computed tomography scans in childhood or adolescence: data linkage study of 11 million Australians. BMJ 346: f2360.

Mettler Jr FA, Wiest PW, Locken JA, Kelsey CA (2000) CT scanning: patterns of use and dose. J Radiol Prot 20: 353-359.

Miglioretti DL, Johnson E, Williams A, Greenlee RT, Weinmann S, Solberg LI, Feigelson HS, Roblin D, Flynn MJ, Vanneman N, Smith-Bindman R
(2013) The use of computed tomography in pediatrics and the associated radiation exposure and estimated cancer risk. JAMA Pediatr 167: 700-707.

Niemann T, Colas L, Roser HW, Santangelo T, Faivre JB, Remy J, Remy-Jardin M, Bremerich J (2015) Estimated risk of radiation-induced cancer from paediatric chest CT: two-year cohort study. Pediatr Radiol 45: 329-336.

NRC (2006) Committee to Assess Health Risks from Exposure to Low Levels of Ionizing Radiation, National Research Council (NRC). Health Risks from Exposure to Low Levels of Ionizing Radiation: BEIR VII-Phase 2. National Academy of Sciences: Washington, DC, USA.

Parkin DM, Darby SC (2011) Cancers in 2010 attributable to ionising radiation exposure in the UK. Br J Cancer 105: S57-S65.

Pearce MS, Salotti JA, Little MP, Mchugh K, Lee C, Kim KP, Howe NL, Ronckers CM, Rajaraman P, Sir Craft AW, Parker L, Berrington de Gonzalez A (2012) Radiation exposure from CT scans in childhood and subsequent risk of leukaemia and brain tumours: a retrospective cohort study. Lancet 380: 499-505.

Pearce MS, Salotti JA, Mchugh K, Metcalf W, Kim KP, Craft AW, Parker L, Ron E (2011) CT scans in young people in Northern England: trends and patterns 1993-2002. Pediatr Radiol 41: 832-838.

PHE (2014) Doses from Computed Tomography (CT) Examinations in the UK-2011 Review. Public Health England: London, UK.

Preston DL, Ron E, Tokuoka S, Funamoto S, Nishi N, Soda M, Mabuchi K, Kodama K (2007) Solid cancer incidence in atomic bomb survivors: 1958-1998. Radiat Res 168: 1-64.

Raelson CA, Kanal KM, Vavilala MS, Rivara FP, Kim LJ, Stewart BK, Cohen WA (2009) Radiation dose and excess risk of cancer in children undergoing neuroangiography. AJR Am J Roentgenol 193: 1621-1628.

Raman SP, Johnson PT, Deshmukh S, Mahesh M, Grant KL, Fishman EK (2013) CT dose reduction applications: available tools on the latest generation of CT scanners. J Am Coll Radiol 10: 37-41.

Richardson DB, Cole SR (2012) Model averaging in the analysis of leukemia mortality among Japanese A-bomb survivors. Radiat Environ Biophys 51: 93-95 discussion 97-100.

Smith-Bindman R, Miglioretti DL, Johnson E, Lee C, Feigelson HS, Flynn M, Greenlee RT, Kruger RL, Hornbrook MC, Roblin D, Solberg LI, Vanneman N, Weinmann S, Williams AE (2012) Use of diagnostic imaging studies and associated radiation exposure for patients enrolled in large integrated health care systems, 1996-2010. JAMA 307: 2400-2409.

Su YP, Niu HW, Chen JB, Fu YH, Xiao GB, Sun QF (2014) Radiation dose in the thyroid and the thyroid cancer risk attributable to CT scans for pediatric patients in one general hospital of China. Int J Environ Res Public Health 11: 2793-2803.

UNSCEAR (2006) Report of the United Nations Scientific Committee on the Effects of Atomic Radiation. UNSCEAR 2006 report. Volume I. Annex A: Epidemiological studies of radiation and cancer. United Nations: New York, USA.

UNSCEAR (2012) Report of the United Nations Scientific Committee on the Effects of Atomic Radiation. UNSCEAR 2012 report. Annex B: Uncertainties in risk estimates for radiation-induced cancer. United Nations: New York, USA.

UNSCEAR (2013) Report of the United Nations Scientific Committee on the Effects of Atomic Radiation. UNSCEAR 2013 report. Volume II. Annex B: Effects of radiation exposures of children. United Nations: New York, USA.

Wakeford R (2013) The risk of childhood leukaemia following exposure to ionising radiation-a review. J Radiol Prot 33: 1-25.

Walsh L, Shore R, Auvinen A, Jung T, Wakeford R (2014) Risks from CT scans-what do recent studies tell us? J Radiol Prot 34: E1-E5.

This work is published under the standard license to publish agreement. After 12 months the work will become freely available and the license terms will switch to a Creative Commons AttributionNonCommercial-Share Alike 4.0 Unported License. 